\title{
8
}

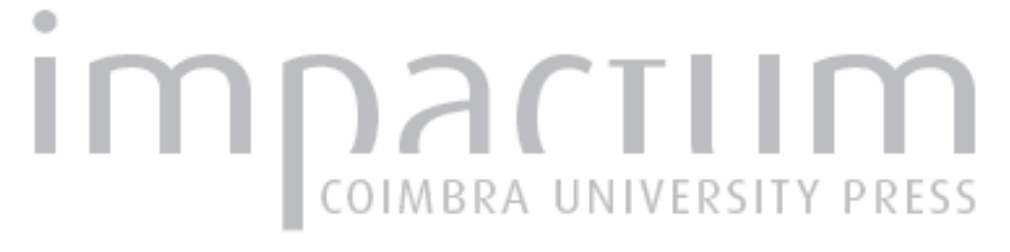

\section{Un nouvel autel découvert à Conimbriga}

\author{
Autor(es): Étienne, Robert
}

Publicado por: Imprensa da Universidade de Coimbra

URL persistente:

URl:http://hdl.handle.net/10316.2/45531

DOI:

DOI:https://dx.doi.org/10.14195/1647-8657_30_9

Accessed : $\quad$ 26-Apr-2023 03:38:54

A navegação consulta e descarregamento dos títulos inseridos nas Bibliotecas Digitais UC Digitalis, UC Pombalina e UC Impactum, pressupõem a aceitação plena e sem reservas dos Termos e Condições de Uso destas Bibliotecas Digitais, disponíveis em https://digitalis.uc.pt/pt-pt/termos.

Conforme exposto nos referidos Termos e Condições de Uso, o descarregamento de títulos de acesso restrito requer uma licença válida de autorização devendo o utilizador aceder ao(s) documento(s) a partir de um endereço de IP da instituição detentora da supramencionada licença.

Ao utilizador é apenas permitido o descarregamento para uso pessoal, pelo que o emprego do(s) título(s) descarregado(s) para outro fim, designadamente comercial, carece de autorização do respetivo autor ou editor da obra.

Na medida em que todas as obras da UC Digitalis se encontram protegidas pelo Código do Direito de Autor e Direitos Conexos e demais legislação aplicável, toda a cópia, parcial ou total, deste documento, nos casos em que é legalmente admitida, deverá conter ou fazer-se acompanhar por este aviso.

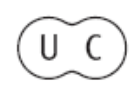


FACULDADE DE LETRAS

INSTITUTO DE ARQUEOLOGIA

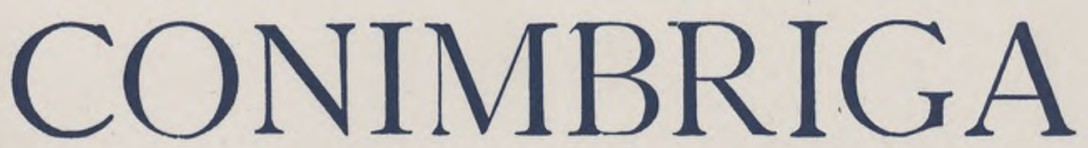

VOLUME XXX

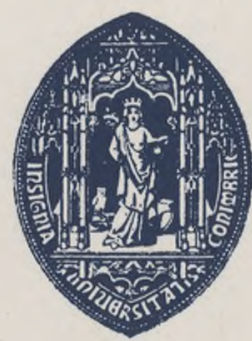

UNIVERSIDADE DE COIMBRA 


\section{ROBERT ÉTIENNE}

\section{Professeur émerite de l’Université de Bordeaux III.}

\section{UN NOUVEL AUTEL DÉCOUVERT À CONIMBRIGA}

“Conimbriga” XXX (1991), p. 121-128

RÉSUMÉ: Un nouveau petit autel a été découvert dans le mur de la basilique chrétienne de Conimbriga. Il est dédié par un indigène, Tanginus, à Jupiter Optimus Maximus, qualifié de C.. Le problème est de savoir comment développer cette abréviation: on peut hésiter entre Conservatori ou Conimbrigensi, sans trouver d'argument suffisant pour choisir entre ces deux solutions qui invoquent, chacune à sa manière, une protection.

Resumo: Descobriu-se urna nova ara na parede da basílica cristã de Conimbriga, dedicada a Júpiter Óptimo Máximo - qualificado de C. — por um indígena, Tanginus.

A questão centra-se, pois, no desdobramento dessa sigla: hesiva-se entre Conservatori ou Conimbrigensi, sem, no entanto, se poderem aduzir argumentos satisfatórios para escolher uma ou outra das soluções, que, apesar de tudo, evocam, cada qual à sua maneira, uma noção de protecção. 
(Página deixada propositadamente em branco) 


\section{UN NOUVEL AUTEL DÉCOUVERT A CONIMBRIGA}

À l'occasion d'un relevé architectural, un nouvel autel a été rencontré par hasard dans le mur de la basilique chrétienne. En entrant dans une série bien connue (') de petits monuments, il présente une dédicace à un Jupiter Optimus Maximus, qualifié de C., abréviation qui fait problème et mérite examen.

Le monument (vidi).

a. Vu de face (fig. 1 A).

Sa hauteur totale, 256 millimètres, se décompose en trois parties: un couronnement $(39 \mathrm{~mm}$ ) où l'on distingue un tympan compris entre deux pulvilli, reposant sur une tablette de 93 millimètres de large. Le tympan est écrêté pour installer la partie circulaire d'un ; une corniche débordant la tablette de 9,1 millimètres de chaque côté, mesurant donc 114 millimètres de large et composé de trois moulures en retrait; le corps de l'autel lui-même définissant un champ épigraphique (129 x 93/92 $\mathrm{mm}$ );enfin le socle fait de trois scoties, en débord l'une sur l'autre et hautes respectivement de 9,5 et 5 millimètres, reposant sur la base proprement dite $(120 \times 40 \mathrm{~mm})$. Les arêtes de la base étant sérieusement épaufrées, il est difficile d'en donner avec précision la largeur.

(') Voir R. ÉtIEnNe, À propos d'une inscription retrouvée de Conimbriga, dans Conimbriga, 29,1990, p. 129-136. 
b. Vu de côté (fig. 1 B).

Tout l'arrière ayant été débité, il n'est pas aisé de mesurer les épaisseurs: celle du fût, par exemple, va de 33 à 46 millimètres, ce qui reste de la corniche et du couronnement ne dépasse pas 36 millimètres et la base est plus épaisse $(55 \mathrm{~mm})$.

c. Vu de dos.

Ayant beaucoup souffert, ce dos ne peut pas nous informer sur la continuation de la corniche et de la base et nous ne savons pas si cet autel était adossé ou non à une paroi.

Quoi qu'il en soit, un tel autel présente une structure et des caractéristiques qui le rapprochent de l'autel L(aribus) Aquitibus, encore que le nôtre présente un focus, qui confirme, si besoin était, sa nature de monument religieux. Taillé dans un calcaire local, friable, provenant du Jurassique inférieur d'Alcabideque, il est aisé à graver et facile à épaufrer, malheureusement. Toutefois il présente un texte complet.

Le texte

Rappelons que ce texte de six lignes occupe la totalité du champ épigraphique.

$\begin{aligned} & \text { I.O.M.C } \\ & \text { TANGI } \\ & \text { NVS.T® } \\ & \text { GINAE.F } \\ & 5 \quad \text { VOTVM } \\ & \text { P.F.S. }\end{aligned}$

Il se développe: $\mathrm{I}($ ovi) $\mathrm{O}$ (ptimo) $\mathrm{M}$ (aximo) $\mathrm{C}$ (développement réservé) Tanginus Tonginae $\mathrm{f}\left(\right.$ ilius ) votum $\mathrm{p}$ (iissime) $\mathrm{f}$ (actum) ( $\left.{ }^{2}\right) \mathrm{s}$ (olvit).

Il se traduit: A Jupiter très bon très grand C [...], Tanginus, fils de Tongina, s'est acquitté de son voeu très pieusement formulé.

La lecture ne bute que sur deux difficultés: à la ligne 3, il faut déceler à l'intérieur du $\mathrm{O}$ un petit $\mathrm{N}$, à peine gravé, haut de 7 millimètres, et à la

${ }^{(2)} \quad$ On pourrait aussi bien penser àp(ia)/(ide) s(olvit): s'est acquitté de son voeu dans un pieux acte de foi. 
ligne 4 un petit $\mathrm{F}$ de 1 centimètre de hauteur. Ce mélange de grandes et de petites lettres représente un tic épigraphique cher à l'atelier de Conimbriga (3). La hauteur moyenne des lettres varie de 1,5 à 1,6 centimètre pour les trois premières lignes, de 1,7 à 1,8 centimètre pour les trois suivantes. A cette régularité qui suppose des lignes de guidage, aujourd'hui invisibles, correspond une mise en page soignée, tant à l'horizontale pour chaque ligne, qu'à la verticale pour l'espacement entre les lignes, soit 5 millimètres, sauf 2 millimètres pour l'espacement entre la dernière ligne et la première moulure du socle. Certes Vordinator n'a pas complètement maîtrisé la distribution de l'espace puisqu'il a recouru à des lettres plus petites; outre le $\mathrm{N}$ et le $\mathrm{F}$ déjà signalés, notons le $\mathrm{M}$ final de la ligne $5(13 / / 14 \mathrm{~mm})$.

La qualité de la gravure est remarquable quand le monument a été bien conservé: le $\mathrm{C}$ de la ligne 1, les $\mathrm{S}$ des lignes 3 et 6 , les $\mathrm{O}$ des lignes 1 et 5 trahissent une main experte et donc nous amènent à conclure en faveur d'une date haute pour cet autel, qu'au plus tard nous attribuerons à la fin du Ier siècle, comme un certain nombre de monuments semblables $\left({ }^{4}\right)$.

\section{Le dédicant.}

Nous avons affaire à un indigène, caractérisé par un nom unique, Tanginus, tout comme son père Tongina. Nous connaissons d'autres individus à Conimbriga qui portent le nom de Tanginus, attesté dans une inscription funéraire $\left(^{5}\right)$ et sur un peson $\left(^{6}\right)$, sans oublier sa présence dans la dèdica $c$ Laribus patriis ( ${ }^{7}$ ). Tanginus et Tongina sont d'origine celtique et typiques de la zone entre Guadiana, Tage et Douro $\left({ }^{8}\right)$. Un nom masculin terminé en - $a$ n'est pas fait pour nous étonner $\left(^{9}\right)$. Nous noterons que la filiation se fait ici non pas par la juxtaposition du nom du père au génitif, déterminant le nom du fils au nominatif, mais par l'intermédiaire de la

(3) Fouilles de Conimbriga, II, p. 215.

(4) Ibid., II, n. ${ }^{\text {ss }}$ 6, 9 (nouvelle datation dans l'article signalé à la n. 1), 10.

(5) Ibid., II, $\mathrm{n}^{\circ} 61$.

(6) Ibid., II, graffite 415 .

${ }^{(7)}$ Ibid., II, appendice I, p. 47-48 = $A E$ 1969-1970, 248, une inscription de Mortágua.

${ }^{8}$ ) J. Untermann, Elementos de un Atlas Antroponimico de la Hispania Antigua (Bibliotheca Praehistorica Hispana, Vil), carte 74, P. 171.

(9) ID., ibid., cartographie (carte 76) Tongeta, p. 174 caractérisant l'aire lusitanienne. 
marque de la filiation par $\mathrm{F}$ (ilius), qui dénote un certain degré de romanisation. Ainsi s'il y a continuité dans l'onomastique indigène d'une génération à l'autre, l'introduction de la filiation par $\mathrm{F}$ est un indice de transformation du milieu local $\left({ }^{10}\right)$. Mais cet indice reste timide de l'adoption d'habitudes romaines: s'agissant d'une inscription votive, est-ce que la dédicace s'adresse à une divinité parfaitement romaine ou à une divinité indigène habillée à la romaine ?

\section{La dédicace.}

La formule finale est à la fois classique votum $\mathrm{s}(\mathrm{olvit})$ et singulière votump(iissime)/(actum) s(olvit) ou/?(ia)/[ide) s(olvit) (n). Il est surtout rare de rencontrer votum écrit en entier ( $\left.{ }^{12}\right)$, ce qui marque l'insistance du dédicant sur l'accomplissement de son voeu, pieusement formulé, ou vœu acquitté dans un pieux acte de foi: la formule insiste sur la religiosité de cet indigène.

A qui s'adresse ce votum ? Au plus grand des dieux, au maître du Panthéon, Jupiter Optimus Maximus. On peut toujours se demander si ce Jupiter si romain et si officiel ne cache pas une divinité indigène qui aurait subi une interpretatio romana, tout comme dans le Nord-Ouest de la Péninsule (13). L'origine celtique du dédicant conforterait cette hypothèse. Comment dans ces conditions développer C. ?

On pourrait songer à trois solutions.

La première consisterait à proposer C(onservatori). Fin 1989, sur 368 inscriptions, entrant dans le corpus épigraphique de Jupiter $\left({ }^{14}\right)$,

$\left(^{10}\right)$ A. Tranoy, La Galice Romaine. Recherches sur le Nord-Ouest de la Péninsule Ibérique dans l'Antiquité (Publications du Centre Pierre Paris, 7), Paris, 1981, p. 368 (= Tranoy, Galice romaine).

(n) L'abréviation ne se rencontre ni dans la liste donnée par R. CAGnat, Cours d'épigraphie latine ${ }^{4}$, Paris, 1914 ni dans les indices des ILS.

${ }^{12}$ ) La formule banale v.s.l.m. se rencontre dans Fouilles de Conimbriga, II, n.os 3, 21; v.s. dans $n^{\circ} 20$; v.s.l.me dans Appendice I, p. 47-48.

$\left.{ }^{13}\right)$ P. LE Roux et A. Tranoy, Rome et les indigènes dans le Nord-Ouest de la Péninsule ibérique. Problèmes d'épigraphie et d'histoire, dans $M C V$, IX, 1973, p. 177-231, surtout p. 218-220; TRANOY, Galice romaine, p. 315-321.

(14) Je remercie Ph. PÉAUd, qui prépare une thèse sur Jupiter dans la Péninsule Ibérique sous la direction d'A. TRANOY, de tous ses renseignements tirés de son fichier informatisé selon le programme PETRAE. 
quatorze seulement associent Conservator soit à Jupiter, soit à lOM. Mais la plupart du temps, Conservator est inscrit en toutes lettres $\left({ }^{15}\right)$ ou abrégé en CONS ( $\left.{ }^{16}\right)$. Cinq inscriptions plus difficiles à interpréter laisseraient une possibilité de développer IOMC en C(onservatori): or l'inscription d'Orense $\left({ }^{17}\right)$, présente IOM à la première ligne et $\mathrm{C}$ à la seconde; cette lettre ne peut être que le praenomen du dédicant, dont les tria nomina sont réduits à leurs initiales. La même condusion devrait être tirée du texte de Covarrubias (Lerma, Burgos) ( $\left.{ }^{18}\right)$, aujourd'hui perdu et sans doute mal transcrit. En tout cas $\mathrm{C}$ serait une initiale de prénom. Seule l'inscription de Lagoaça (Freixo de Espada à Cinta, Bragance) $\left({ }^{19}\right)$ pourrait fournir la possibilité de développer $\mathrm{C}$ en $\mathrm{C}$ (onservatori), puisque la lettre intervient sur la même ligne que IOM. On pourrait joindre à ce texte deux autres inscriptions portugaises: 1'une à Carviçais $\left({ }^{20}\right)$, une autre à Sabrosa do Douro (21).

La seconde possibilité envisagerait de comprendre C(ustodi). Or l'inscription d'Astorga (Asturica) (22) présente Custodi en toutes lettres sur une seule ligne; donc développer $\mathrm{C}$ en Custodi serait bien hasardeux, d'autant que le contexte de l'inscription nous contraint d'y voir un culte officiel et capitolin.

Le même raisonnement nous conduit à abandonner une troisième possibilité où $\mathrm{C}$ serait l'initiale de Capitolino, car l'inscription de Tarragone ${ }^{(23}$ ) offre l'épithète complète, sans abréviation.

Ainsi jusqu'à présent la statistique nous invite à opter pour un Jupiter Optimus Maximus Conservator. Toutefois, puisque nous sommes à Conimbriga, comment ne pas penser à un Jupiter Optimus Maximus Conimbrigensis, faisant de Jupiter un dieu tutélaire de la cité ? Nombreux sont les exemples où $I O M$ est lié au processus de municipalisation, tel IOM Municipalis à Chaves $\left({ }^{24}\right)$. Les communautés semi-urbaines -

(15) Comme par exemple dans IRPL, 4 où Conservatori occupe deux lignes de l'inscription.

(16) $A E$ 1924, 11; AF 1976, 321.

(17) IRG, IV, 114.

(18) CIL II, 2850.

(19) TAE, 1977, p. 167-175.

(20) $A P$, Vili, 1903, p. 156.

(21) $A E 1985,574$.

(22) $A E 1968,229$.

(23) CIL II, $4079=$ RIT, 33.

(24) $A E 1973,305$. 
centuries ou castella $\left({ }^{25}\right)$ - font aussi des dédicaces à IOM, qui joue un rôle fédérateur avant le développement des villes à l'époque flavienne. Certaines dédicaces $\left({ }^{26}\right)$ ont été également retrouvées dans les temples des castros ou à proximité, comme à São Mamede (Moncorvo), où $I O M$ est associé à la civitas Baniensis $\left({ }^{27}\right)$ : on y retrouve le rôle joué par Jupiter, protecteur de la cité. À Conimbriga, sur un autel d'époque flavienne $\left({ }^{28}\right)$, sont exaltés les Lares de Flavia Conimbriga: la population du jeune municipe ressent le lancinant besoin de faire appel à leur tutèle, tout comme un cavalier de la III e cohorte des Lusitaniens $\left({ }^{29}\right)$ s'est acquitté d'un voeu auxDiis Deabusque Conimbric (ensibus ou ensium). Les divinités protègent la communauté humaine habitant l'oppidum de Conimbriga, et le lieu lui-même; sous leur anonymat elles rassemblent toutes les forces divines qui peuvent concourir à leur protection. Elles englobent les divinités indigènes en train de se romaniser et sans doute aussi celles du panthéon romain. Pourquoi, dans ces conditions, ne pas comprendre le nouveau texte de Conimbriga comme s'adressant à un Jupiter Optimus Maximus Conimbrigensis, d'autant qu'il émane d'un indigène à l'onomastique typiquement lusitanienne ? Avouons cependant nos hésitations. Conimbricenses qualifie en toutes lettres les $D U$ et les Deae, et de plus à une époque certainement antérieure à notre nouveau document.

Dans ces conditions nous ne pouvons qu'avouer la belle incertitude du développement des sigles. Les deux développements trahissent en tout cas la même spiritualité, celle de l'invocation d'une protection, l'une relevant d'une forme plus romaine, conservatori, l'autre, conimbricensi, accentuant le caractère indigène qui s'imposerait avec l'onomastique du dédicant et celle de son père. L'interpretatio romana se ferait alors moins brutale que dans le premier cas et ménagerait sous les Flaviens les transitions vers une totale romanisation.

(25) Actas del II Seminario de Arqueología del Noroeste, p. 172,173; AF 1928, 162.

(26) CIL II 2850 et AF 1976, 321.

(27) CIL II, $2399=$ EE Vili, $107=$ EE IX $98=I L E R, 47$.

${ }^{(28)}$ Fouilles de Conimbriga II, $\mathrm{n}^{\circ} 10$.

$\left.{ }^{29}\right)$ Ibid, n ${ }^{\circ} 3$. 


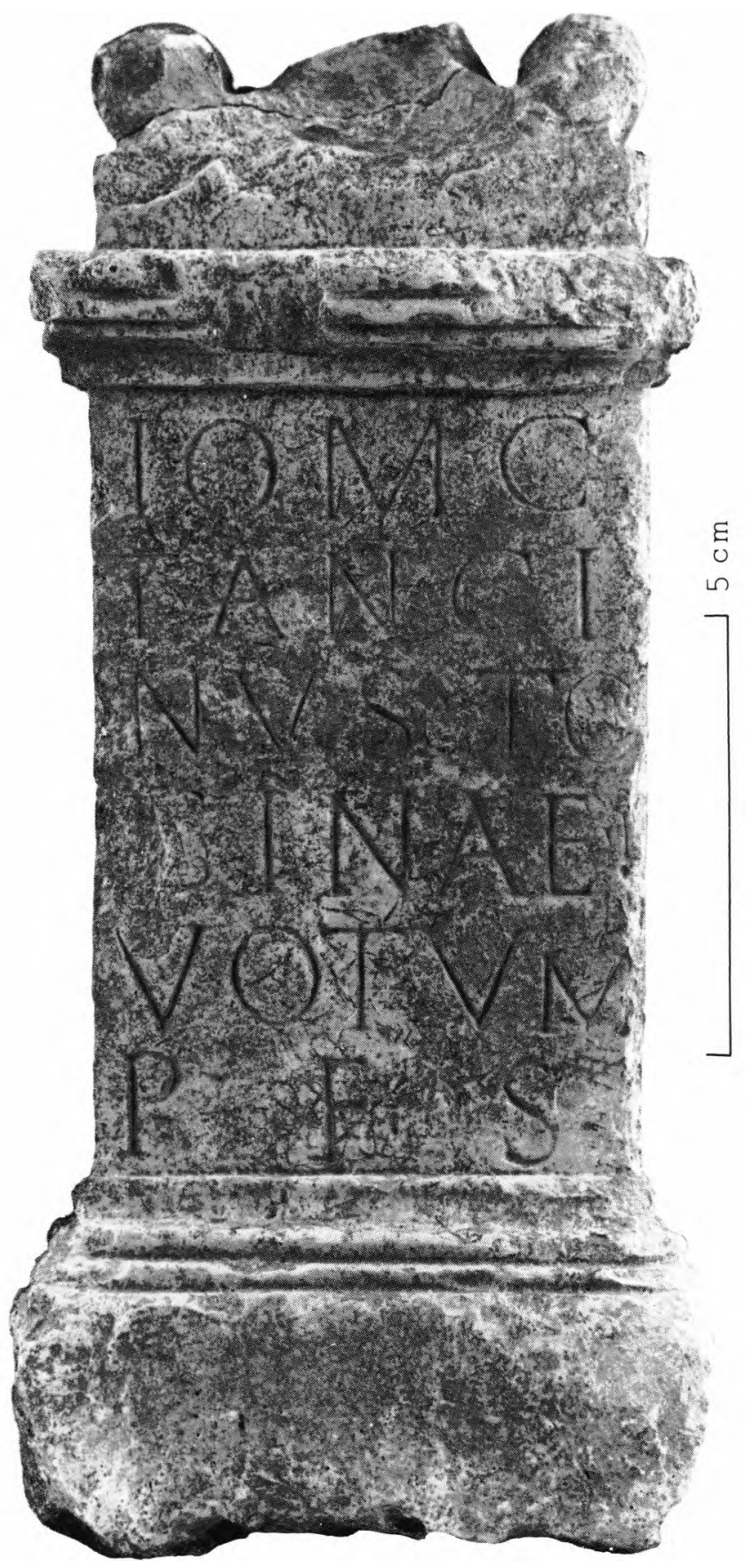

FIG. A 


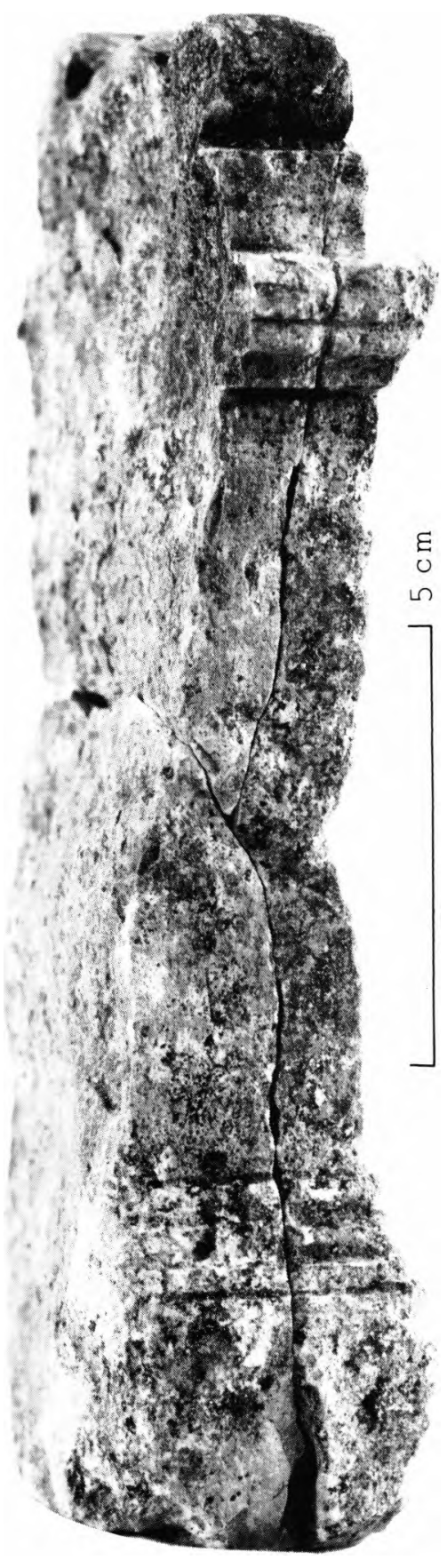

FIG. B 